\title{
Clarifying consciousness
}

\section{Work is under way to relate the mind to observable neurobiological events.}

\section{The Quest for Consciousness: A Neurobiological Approach \\ by Christof Koch \\ Roberts: 2004. 448 pp. \$45, £29.99}

Wider than the Sky: The Phenomenal Gift of Consciousness by Gerald M. Edelman

Yale University Press: 2004. 185 pp. \$24

\section{Jean-Pierre Changeux}

The relationship between body and soul has been fiercely debated since Ancient Greece, first by philosophers and then by scientists. But until the 1980s it was not considered 'scientifically correct' for physiologists to mention the word 'consciousness' in a refereed paper. Christof Koch's book The Quest for Consciousness shows that this period is definitively over. The many facets of consciousness that philosophers have put forward are still not yet understood, but Koch's book represents an exceptionally rich presentation of existing experimental work, accompanied by many cogent, if still informal and debatable, theoretical statements.

From the start, the reader is taken on an in-depth exploration of the most recent developments in the biology of consciousness, guided by Koch and Francis Crick, with whom Koch has collaborated for the past 20 years. The outcome is, in myview, exceptional.

This book and a few others, in particular Gerald Edelman's Wider than the Sky, also reviewed here, bring us to the stage where, in my opinion, objective physiological recordings and measurable physical parameters can be causally linked with subjective experience. This is a strong statement. Koch is more cautious, stating that his quest is for the neuronal correlates of consciousness. His aim is to establish an "explicit correspondence" between mental and neuronal events. But, in my opinion, causal determination, despite all its difficulties, is required. Koch's position therefore seems less bold than that set out in Crick's own book The Astonishing Hypothesis (Simon \& Schuster, 1994): "You are in fact no more than the behaviour of a vast assembly of nerve cells and their associated molecules."

The explosive development of new methods of brain exploration, in particular brain imaging, as well as imaginative cognitive experimentation, has created an important experimental literature on consciousness, which Koch's book covers in a vivid and fluent style. He has deliberately not covered some important issues, such as the selfreferential aspect of consciousness and the role of language and emotions. Instead, the focus is on perception, particularly visual

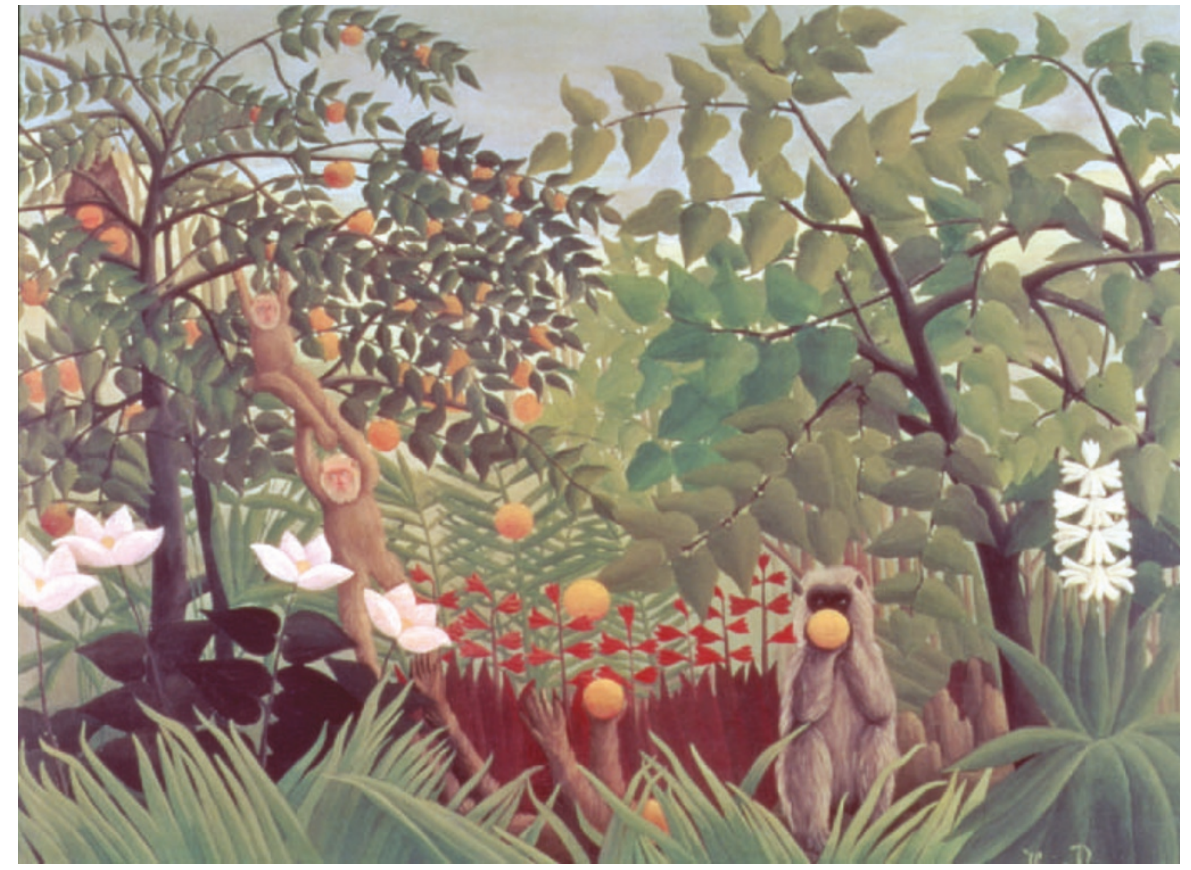

The 'exotic landscape' of our perceptions, reflected in this painting by Henri Rousseau.

perception. Koch traces the diverse routes from the retina through the visual cortices to the frontal cortex and then to the motor areas, defining step by step the necessary conditions for the neuronal correlates of consciousness. This bias towards an input-output view of information processing neglects the role of spontaneous states of neural activity and contrasts with the top-down projective style of the brain, which constantly anticipates the evolution of the outside world - this should, in my opinion, be an essential part of any theory of consciousness.

Perhaps Koch's most controversial proposal is that "zombies could be living among us". According to Crick and Koch, these fictitious creatures would be "devoid of any conscious experience" and yet would have behaviour identical to that of their normal, conscious counterparts. This can only be a metaphor, not a serious model, yet it might help to illustrate the notion that specialized sensorimotor reflex processes (referred to as 'zombie agents') carry out routine missions in the absence of any direct conscious sensation or control.

This concept is reminiscent of Pierre Janet's "subconscious actions", as presented in his 1889 book L'automatisme psychologique, which are "entirely absent from the vision of the subject, thus forcing ourselves to assume their existence outside consciousness". Janet, like Koch, based his argument on a clinical framework, including catalepsy, somnambulism, hallucinations and anaes- thesia. It is fascinating to follow Koch's presentation of the latest clinical observations, together with abundant psychophysical experiments, in which reasonable attempts have recently been made to uncover neural signatures for conscious and non-conscious processing. Examples include the views from functional magnetic resonance imaging (fMRI) of the masking experiments of Stanislas Dehaene and colleagues, and Nikos Logothetis' dynamic electrophysiological recordings and fMRI images from binocularrivalry experiments.

Koch also discusses the idea that the perception of motion consists of discrete processing epochs, or snapshots. He reexamines the classical paradox of the continuity of cinematographic vision, recently refreshed by Oliver Sacks' clinical observations of patients who see movement as a succession of still images during migraine episodes. This rather simple experimental paradigm deserves to be re-explored using the tools available today. Understanding this, as well as many of the other issues raised by The Quest for Consciousness, should tell us more about the access to, and persistence of, visual stimuli in consciousness.

Koch postulates that if perceptual representations are to achieve conscious status, they must be intimately linked to an intentional planning stage. He therefore introduces a requirement for projection to the front of the brain. Nevertheless, Crick and Koch follow Ray Jackendoff in postulating 
that many aspects of high-level cognitive functions, such as decision-making, planning and creativity, are themselves hidden from consciousness: "You don't know your innermost thoughts." These thoughts are carried by "a little person, a homunculus, inside my head who perceives the world through the senses, who thinks, who plans and carries out voluntary actions" - another metaphor. This position implies that "you are aware only of the sensory representations associated with these mental activities."

This is reminiscent of a debate in the nineteenth century about the role of images in thought. In 1870, Hippolyte Taine compared the mind to "a polype of images", and recent ingenious experiments by Gordon Shepard and Stephen Kosslyn have confirmed the role of mental imagery as central to the substance of conscious thought. Yet it seems highly debatable that all the contents of consciousness are sensory. What about mathematical concepts and their creation? What about consciousness of our errors? It seems to me that many abstract non-sensory representations can also be conscious. But Koch's speculation does bring forward an important question: can we define the particular kind of conscious and non-conscious representations to which the prefrontal cortex contributes? I am convinced that these issues can now be investigated in neurobiological terms.

An important quality of The Quest for Consciousness is the book's attempt to compare the views of Koch and Crick to the work of others - in particular to Edelman's sophisticated framework, which is elegantly summarized in Wider than the Sky. Koch's competition between coalitions of neurons fits well with Edelman's clusters of neurons and group selection by a massive feedback signalling loop. However, Edelman's position contrasts in another respect with that of Crick and Koch (and Dehaene and myself, for that matter), who posit well-defined neural architectures for consciousness. These structures appeared in the course of evolution - possibly from what Derek Denton refers to as "primal emotions", such as thirst, hunger and sex - and relate in particular to the expansion of the prefrontal cortex and its inhibitory power. A further distinction between the view of Crick and Koch and that shared by Dehaene and myself is our emphasis on neural mechanisms of evaluation for actualized actions, but also self-evaluation for tacit plans. This crucial mobilization of reward systems in consciousness is missing from Koch's book.

In a field that is plagued by more philosophical than scientifically sound controversies, Koch's book is, on the whole, remarkably balanced. But it does not touch on one crucial issue: can a model of consciousness be formalized in mathematical terms? Is it realistic to conceive a computer model or a robot that implements the conscious versus non-conscious processing of sensory stimuli? My own answer would be a resounding 'yes'. I hope that the next generation of neuroscientists, inspired by reading The Quest for Consciousness, will soon start planning their experiments.

Jean-Pierre Changeux is at the Molecular

Neurobiology Laboratory, Institut Pasteur,

28 rue du Docteur Roux, 75724 Paris, France.

\section{Keep it simple}

Deep Simplicity: Chaos, Complexity and the Emergence of Life

by John Gribbin

Allen Lane: 2004. 272 pp. 18.99

Mark Buchanan

The search for simplicity is perhaps the most basic theme of all science. As the late social and political scientist Herbert Simon put it, the purpose of science is "to find meaningful simplicity in the midst of disorderly complexity". In his new book Deep Simplicity, John Gribbin explores this theme in the context of two great movements of modern science - chaos and complexity and argues that the discovery of simplicity hiding behind surface complexities will soon explain the origin of life itself.

Gribbin suggests quite plausibly that humans - and, by implication, our societies - are among the most complex things in the Universe. At the atomic level, individual particles follow relatively simple physical laws. It is out of the interactions of many particles, and then of objects made of them, that complexity arises, producing conductors and liquid crystals, biomolecules, living organisms, ecosystems and human culture. On a larger scale, the world again becomes relatively simple, for in the interior of a large planet, or a star, "gravity crushes any structure out of existence". Complexity occupies a middle world, which is also, probably for good reason, our world.

The aim of the book is to explore how simplicity arises on this level, and how it can be identified. But first Gribbin establishes why complexity, or at least the appearance of complexity, should be expected.

The classical newtonian view of the fully predictable Universe dominated science for two centuries. But as Gribbin points out, this world view actually rested on a vast leap of faith - on the supposition that if scientists were clever enough to solve Newton's equations for any system of interacting particles, their solutions would be just as regular as the periodic motion of two bodies, reflected in the elliptic orbits of the planets about the Sun. In 1890, the French mathematician Henri Poincaré proved otherwise: that the resulting motion can be irregular and unpredictable, even when only three bodies are involved. "It may happen," Poincaré wrote, "that small differences in the initial conditions produce very great ones in the final phenomena." This, in modern parlance, is chaos, and it implies - in the more general context of dynamical-systems theory - that scientific prediction over long periods of time is generally impossible.

Gribbin tells the story of the modern rediscovery in the 1960s and 1970s of Poincaré's insight. This is an exciting tale but has been told before, most notably in James Gleick's bestseller Chaos (Heinemann, 1988). On the positive side, the discovery of chaos reveals that many highly erratic phenomena, ranging from chemical reactions to fluctuations in biological populations, may actually arise from very simple underlying dynamics. This is one way that simplicity often lies behind complexity.

Gribbin then weaves the story of chaos together with more recent developments, and with a host of topics now gathered together under the term 'complexity science'. The book moves rapidly from spontaneous pattern formation to the mathematics of fractals and the idea of self-organized criticality, examining its relation to earthquakes, mass extinctions and a vast range of other prominently unpredictable phenomena. The book celebrates the contemporary emphasis, especially in physics, on seeking the explanation of complex phenomena through simple dynamical models of growth and evolution. The lesson is the same everywhere: what appears as surface complexity often has its origins in dynamical simplicity. Importantly, Gribbin points out that modern computers have played a central role in making the complexity sciences possible, altering not only the content of science but the way it is done.

Much of Deep Simplicity will be familiar to anyone who has read about chaos and complexity before, but Gribbin does his usually excellent job of making complicated ideas accessible to a broad readership, and the book would certainly make an excellent non-technical introduction to this way of thinking. One minor shortcoming is that the book could have been written in, say, 1998, and still contained virtually all the same material. This is a little disappointing, as the past five years have witnessed a flowering of the complexity sciences and their successful application to a broad range of scientific topics.

Gribbin is something of a phenomenon of science writing, judging from his prolific output over the past two decades. In Deep Simplicity, perhaps, he doesn't quite succeed in showing how chaos and complexity will soon "explain the origin of life itself". But he breathes life into the core ideas of complexity science, and argues convincingly that the basic laws, even in biology, will ultimately turn out to be simple.

Mark Buchanan is a freelance writer based in the United Kingdom. 